\title{
Power of dissipation in a rotating machine
}

\author{
Pavel Žitek ${ }^{1, *}$, Marek Klimko ${ }^{1}$, and Tomáš Noga ${ }^{1}$ \\ ${ }^{1}$ University of West Bohemia in Pilsen,Univerzitní 8, 30614 Pilsen, Czech Republic
}

\begin{abstract}
This article deals with a possibility of how to determine a power dissipation in a rotating machine. It divides losses in three parts according to their origin - losses arising around the rotating disc, in bearings, and by a friction of a shaft surface due to viscosity. A precise determination of the power dissipation is important when evaluating the efficiency of the whole device. The procedure is graphically illustrated on the example of a single-stage experimental turbine.
\end{abstract}

\section{Introduction}

It is important to know dissipations emerging in a rotor to properly determine a rotating system power. We will demonstrate a procedure of determination of this power on the rotor of the air turbine VT-400.

The core is to detect the power of the turbine stage to determine its efficiency. (1). The power can be determined from the dynamometer connected to the turbine rotor. However, the dynamometer power is lower by the dissipation power, compared to the stage power, therefore it has to be included in the calculation of the efficiency

$$
\begin{gathered}
\eta_{o b v}=\frac{\frac{P_{s t}}{m_{v}}}{h_{0}-\Delta h_{c 2}} \quad[1] \\
\eta_{o b v}=\frac{\frac{P_{D}+P_{f}}{m_{v}}}{h_{0}-\Delta h_{c 2}} \quad[1]
\end{gathered}
$$

where

$\begin{array}{ll}\eta_{o b v} & \text { stage efficiency } \\ P_{s t} & \text { stage power } \\ m_{v} & \text { air mass flow rate } \\ h_{0} & \text { isoentropic drop on the stage } \\ \Delta h_{c 2} & \text { kinetic energy in the air leaving the stage } \\ P_{D} & \text { power obtained from the dynamometer } \\ P_{f} & \text { dissipation power }\end{array}$

Furthermore, we will consider dissipations caused by a friction of the disc, bandage, shaft and bearings.

\section{Disc friction dissipation power}

Literature mentions various mathematical relations to calculate the friction dissipation of rotating discs. Basically, they are a result of a dual approach to this problem. One group of equations originates from results of experiments, whereas the second one applies the basic laws of fluid mechanics on a rotating disc friction in the environment.

\subsection{Experimental research}

The mutual sign of equations obtained experimentally is almost the same functional dependency of dissipations on the peripheral velocity (revolutions) and the disc diameter. Yet, the constants in the equations differ, due to the fact that experiments have been carried out with discs of a different thickness; distances of limiting walls were different as well, both in the axial and radial directions. Another difference lies is a different value of Reynolds number during measurements. Higher values of constants correspond to small $\mathrm{Re}$ values and vice versa.

Generally, all relations (except one) can be written in the following form:

$$
P_{f d}=k \cdot\left(\frac{u}{10}\right)^{3} \cdot d^{2} \cdot \rho \quad[W]
$$

where:

$d \quad$ disc diameter

$u \quad$ peripheral velocity

$k$ coefficient resulting from a particular experimental research

We have focused on four authors out of all studied approaches.

The first is prof. Stodola [1]. He determined the coefficient $k=1$ (for superheated steam) and $k=1.25$ for saturated steam. Based on experiments carried out later on, prof. Stodola defined the coefficient $k=2.7$.

The next author is Zalf [2]. He determined the coefficient oscillating within the range of $k \in$ $\langle 0.865,1.73\rangle$, depending on the distance of limiting surfaces. The bottom boundary of the coefficient applies for small distances from limiting surfaces, upon increasing the distance the coefficient value grows. The coefficient values have come out of precise

\footnotetext{
Corresponding author: zitek@,kke.zcu.cz
} 
measurements on real turbine stages within the area of $R e>5 \cdot 10^{6}$, whereas Reynolds number for the disc is defined:

$$
R e=\frac{d \cdot u}{v}
$$

where $v$ is kinematic viscosity of the environment surrounding the rotating disc.

The third reviewed author is Scegliaev [3]. The equation, unlike the others in this group, includes also a friction dissipation of a bandage connected to moving blades by rivets with smaller heads. He defines the coefficient as $k=44$.

The last research we have studied was led by prof. Miskovsky [4]. He presents the equation in a slightly different form:

$$
P_{f d}=k \cdot\left(\frac{u}{10}\right)^{2.8} \cdot d^{1.4} \cdot \rho \quad[W]
$$

The coefficient value varies within the range of $k \in\langle 0.08,0.1\rangle$.

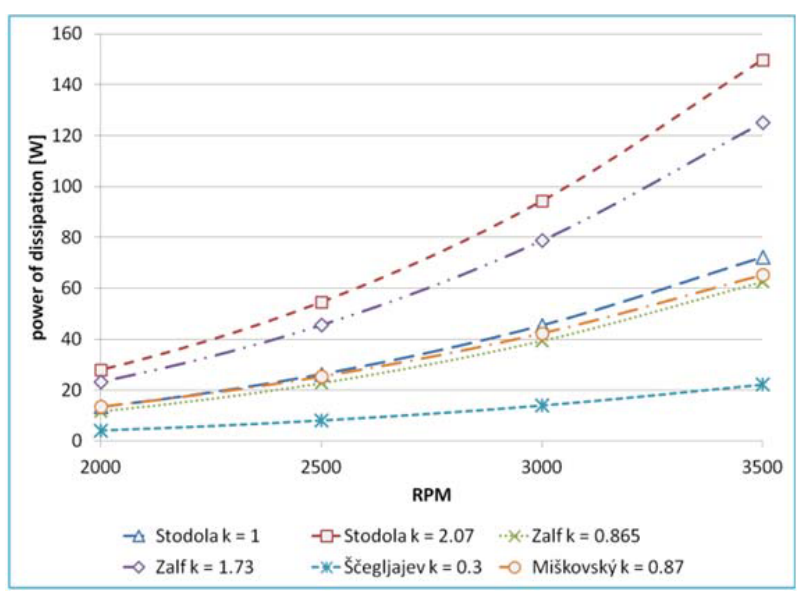

Fig. 1 The course of dissipations caused by the turbine VT -400 disc friction according to experiments

\subsection{Basic laws of fluid mechanics}

The second group of equations results from the basic laws of fluid mechanics, which are applied in order to determine the friction resistance and its moments acting on the rotating board in the environment of density $\rho$. The relevant equations contain generally the disc friction coefficient, which is dependent either on Reynolds number only or also on the proportional distance of limiting walls from the disc. The results can be summarized in one general relation:

$$
P_{f d}=k \cdot c_{t} \cdot u^{3} \cdot r^{2} \cdot \rho[W]
$$

where:

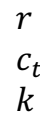
coefficient defined by particular authors
According to Traupel [5], $c_{t}$ is defined as follows (for $R e>2 \cdot 10^{5}$; whereas the Re number definition is according to the equation (4)):

$$
c_{t}=\frac{0.003}{R e^{0.2}}
$$

According to Scegliaev [3], the friction coefficient for turbulent flow within the disc area is

$$
c_{t}=2.5 \cdot 10^{-2} \cdot\left(\frac{S}{r}\right)^{0.1} \cdot R e^{-0.2}
$$

where $s$ is the distance of the limiting wall from the disc.

According to Tuliszky [6], the friction coefficient is again (beside others) a function of Reynolds number. However, it is a function of the radius instead of the diameter in this case.

$$
c_{t}=\frac{C_{R}}{R e^{0.2}}=\frac{C_{R}}{\left(\frac{u \cdot r}{v}\right)^{0.2}}
$$

where $C_{R}$ is so-called reduced disc friction coefficient depending on the proportional distance $s / r$ (fig. 2).
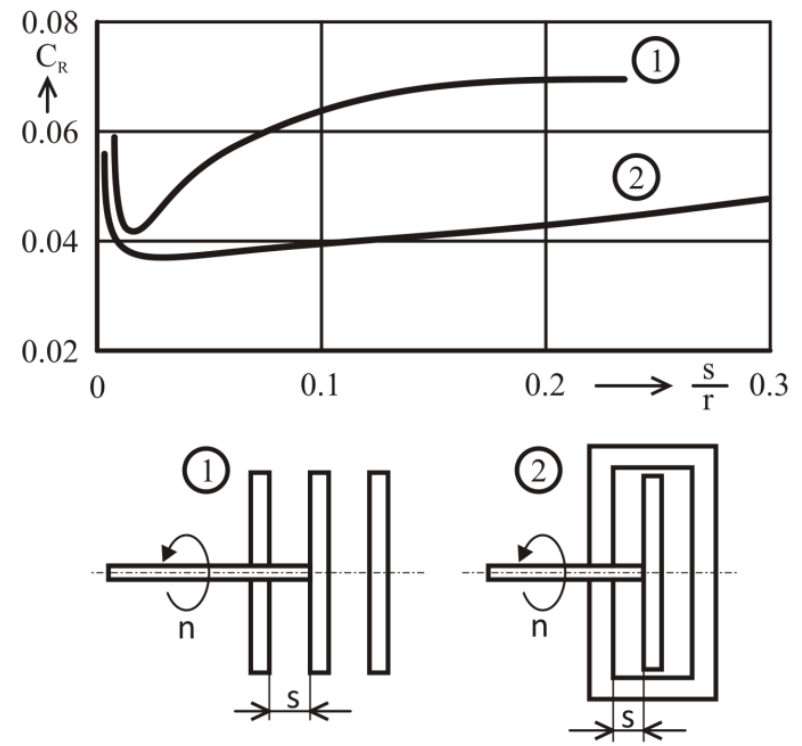

Fig. 2 Reduced disc friction coefficient

Linnecken [7] has provided a very detailed study. He has deduced the following relation for the friction coefficient:

$$
c_{t}=\frac{0.5 \cdot \pi}{\frac{s}{r} \cdot \operatorname{Re}}+\frac{0.04}{R e^{0.2}} \cdot \frac{1+\frac{s}{r}}{2+\frac{s}{r}} \mid R e=\frac{u \cdot r}{v}
$$

The dissipation power is a function of a peripheral velocity, a disc diameter, the friction coefficient and the coefficient $k$ in all four cases. However, that is the only compliance. As demonstrated above, the difference is not only in the coefficient $k$, but also in the disc friction coefficient $c_{t}$. Traupel, for instance, doesn't take the 
limiting surfaces influence into consideration. Nevertheless, the ratio $s / r$ is of a not inconsiderable influence, according to the remaining three authors.

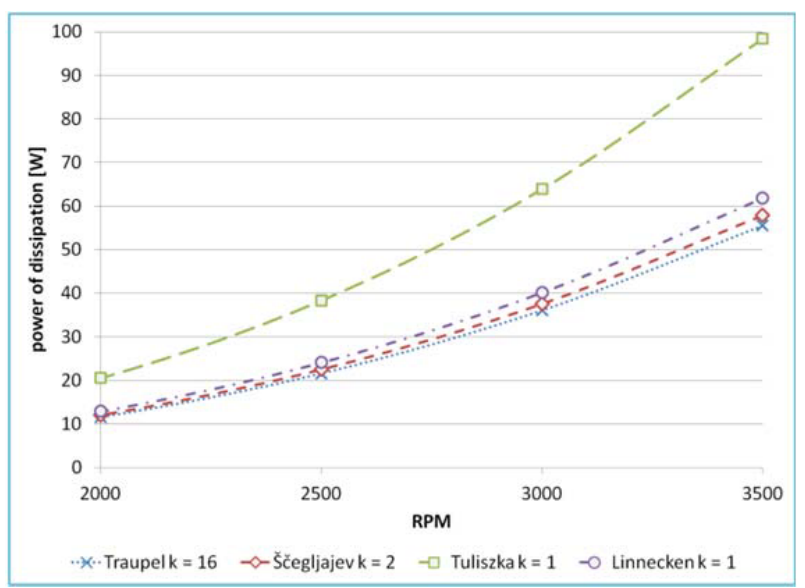

Fig. 3 The course of dissipations caused by the turbine VT - 400 disc friction according to fluid mechanics

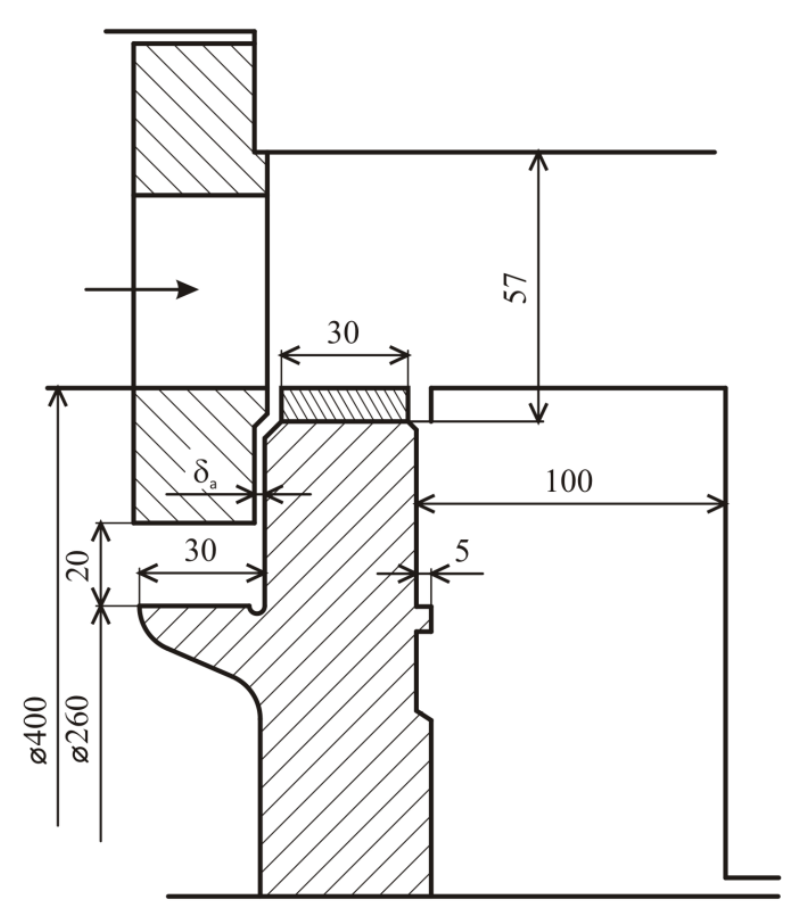

Fig. 4 Scheme of the stage for a friction dissipation calculation

The equations obtained by both approaches have naturally been applied on VT-400 (fig. 1, fig. 3). We have chosen the ratio $s / r=1$ given the geometry of the flow part of the turbine (fig. 4).

\subsection{Summary}

Taking into account the conditions used by individual authors, limiting walls influence, the flow part geometry and other circumstances, we have chosen the relation deduced by Linnecken (6), (10). The course of the dissipation power is presented in fig. 5 .

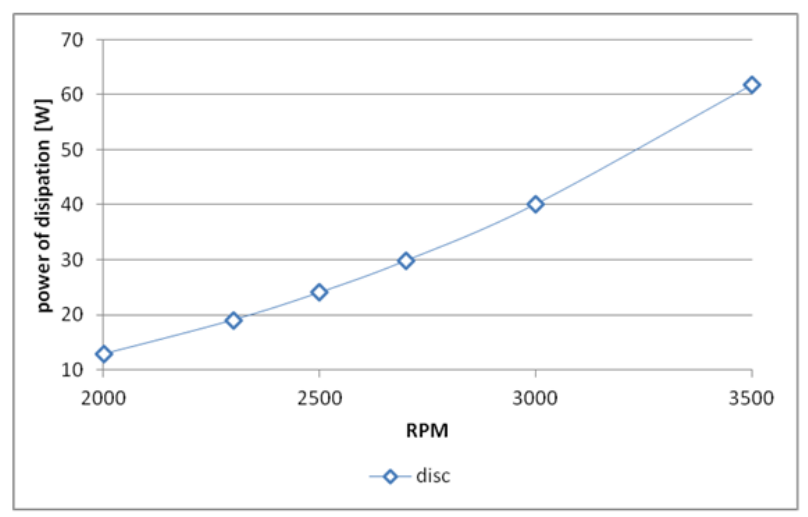

Fig. 5 Disc dissipation power

\section{Dissipation power caused by the bandage friction and projections on the disc}

\subsection{Bandage}

The most detailed calculation of the bandage friction dissipation is mentioned by Linnecken again:

$$
P_{f b}=c_{t} \cdot u_{b}^{3} \cdot r_{b} \cdot b \cdot \rho[W]
$$

where:

$\begin{array}{ll}u_{b} & \text { bandage peripheral velocity } \\ r_{b} & \text { bandage diameter } \\ b & \text { disc width }\end{array}$

$c_{t}=\frac{2 \cdot \pi}{\frac{t}{r_{b}} \cdot R e_{b}}+\frac{0.15}{R e_{b}^{0.2}} \cdot \frac{1+\frac{t}{r_{b}}}{2+\frac{t}{r_{b}}} \mid \operatorname{Re}=\frac{u_{b} \cdot r_{b}}{v}$

where:

$t$ distance of the limiting wall from the bandage

As evident (12), $c_{t}$ is a function of the flow part geometry and Reynolds number. As a matter of interest, we are adding the geometry dependency of $c_{t}$ for different values of Reynolds number, see fig. 6 .

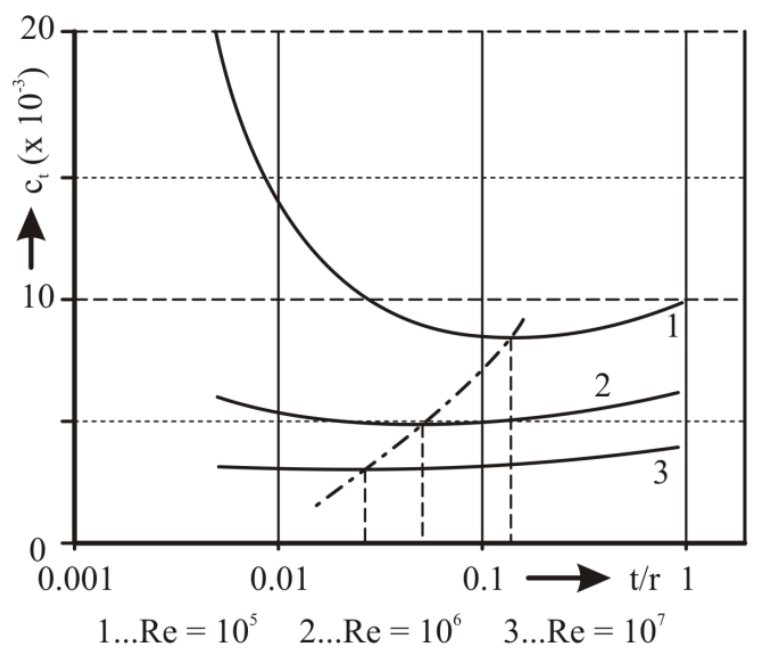

Fig. 6 Dependence of $\boldsymbol{c}_{\boldsymbol{t}}$ on the bandage geometry 


\subsection{Projections on the disc}

Another type of dissipation taken into account are these caused by the friction of projections in the surrounding environment. The calculation is entirely the same as for bandage dissipation power, yet the difference is in substituted values, which is apparent in fig. 4. The resulting value of the dissipation power is lower with respect to smaller diameter of the projection, leading to a lower peripheral velocity, and also given a larger distance from limiting surfaces (see fig. 7)

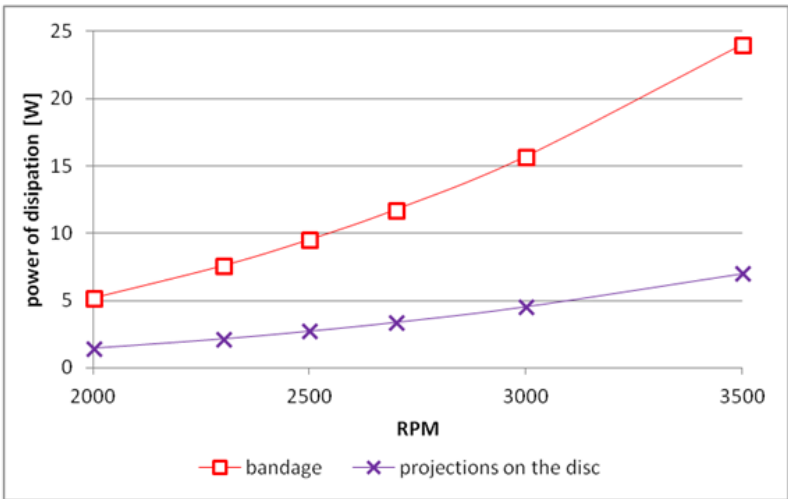

Fig. 7 Dissipation power of the bandage and projections on the disc

\section{Dissipation power in bearings}

This chapter will deal with the second part of the total friction losses, particularly dissipations in bearings. These losses can be determined by a calculation and are given by the following relation:

$$
P_{L}=\frac{\pi \cdot d \cdot n \cdot F_{e} \cdot f_{t}}{60} \quad[W]
$$

where:

$d \quad$ inner diameter of a bearing $f_{t} \quad$ rolling friction coefficient (single row ball bearings $f_{t}=0.0015$, tilting double row bearings $f_{t}=0.002$ )

$F_{e} \quad$ equivalent force in a particular bearing

\subsection{Axial force}

Axial force acting on bearings is defined by the difference of pressures in front of and behind a blade wheel. It means that it is a function of the stage reaction $\rho$, chosen $u / c$ (since $c$ is defined by the pressure drop) and also the speed $n$ (it is necessary to change $\Delta p$ along with the change of speed to maintain optimal $u / c$ ).

Therefore, $F_{A}=f(\rho, u / c, n)$.

As the determination of axial dissipations depends on several variables, their determination can be proceeded only for concrete conditions set by experimental measurements.

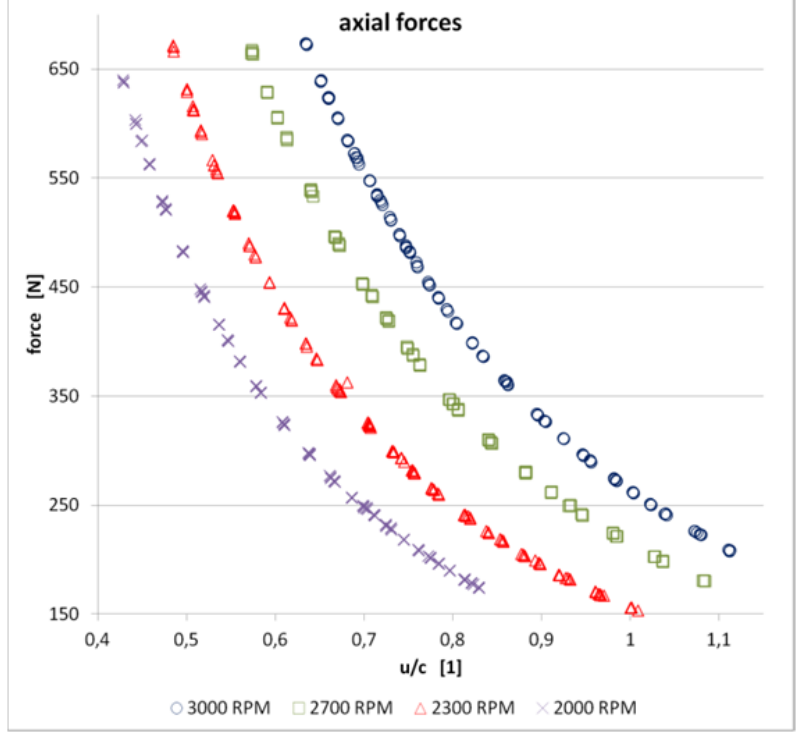

Fig. 8 Influence of $\mathrm{u} / \mathrm{c}$ and speed on axial force

\subsection{Radial forces}

We will use the scheme in fig. 9 to count up reaction forces in bearings.

We will calculate radial forces in bearings, i.e. reactions $R_{A}$ and $R_{B} \mathrm{Z}$, from the equilibrium of forces and moments. We will use the detected radial and axial forces to calculate so-called equivalent force acting on the bearing.

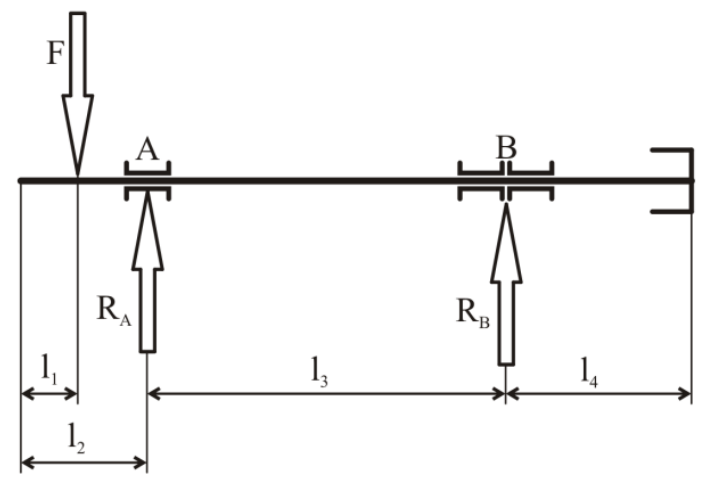

Fig. 9 Scheme of the shaft

$$
\begin{gathered}
\sum F_{i x}=0 \\
\sum F_{i y}=0: R_{A}-F-q \cdot l+R_{B}=0 \\
\sum F_{i}(B)=0: R_{A} \cdot l_{3}- \\
-F \cdot\left[l_{3}+\left(l_{2}-l_{1}\right)\right]- \\
-q \cdot \frac{\left(l_{2}+l_{3}\right)^{2}}{2}+q \cdot \frac{l_{4}^{2}}{2}
\end{gathered}
$$

$R_{A}=\frac{F\left[l_{3}+\left(l_{2}-l_{1}\right)\right]+q \frac{\left(l_{2+l_{3}}\right)^{2}}{2}-q \frac{l_{4}^{2}}{2}}{l_{3}}$ 


\subsection{Equivalent force}

Equivalent force can be determined several ways. Three of them are listed as follows:

Niemann determines the equivalent force

$$
F_{e}=F_{R}+2.75 F_{A}
$$

Fronius considers

$$
F_{e}=\sqrt{F_{R}^{2}+F_{A}^{2}}
$$

The equivalent force according to Swen

$$
F_{e}=X_{o} \cdot F_{R}+Y_{o} \cdot F_{A}
$$

Apart from $F_{A}$ and $F_{R}$, there are also coefficients $X_{0}$ and $Z_{0}$ appearing in the relation (21). We assume the front tilting double row bearing (fig. 10), with an angle $\alpha=10^{\circ}$ and relevant coefficients $X_{0}=1, Y_{0}=2.6$, to capture the whole axial force. An example can demonstrate that dissipations in bearings are progressively $54.21(19)-41.41(20)-53.5$ (21) watts instead of 41.09, not considering the axial force, for revolutions of $4000 \mathrm{~min}^{-1}$, according to the above mentioned authors. As apparent, the axial force has basically no influence on the equivalent force in Fronius's relation. We have use Niemann's relation for our computation.

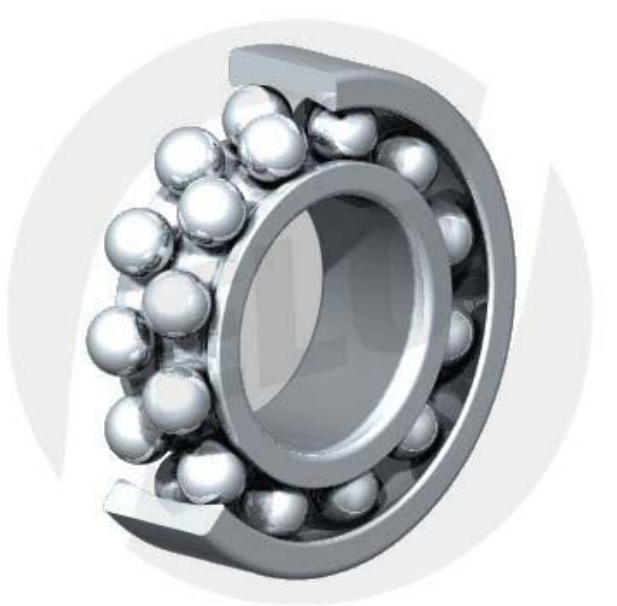

Fig. 10 Tilting double row bearings [8]

\subsection{Summary of dissipations in bearings}

As already presented, axial force changes depending on several variables, whereas the radial force is determined by the rotor geometry, or its weight. Should we consider one version of blading (i.e. steady reaction stage) and measure using the same $u / c$, we will obtain different axial forces for different revolutions (see table 1).

Table 1 Axial and radial forces

\begin{tabular}{|l|c|c|c|c|}
\hline rpm & 2000 & 2300 & 2700 & 3000 \\
\hline$F_{A}[N]$ & 260 & 346 & 479 & 580 \\
\hline$R_{A}[N]$ & \multicolumn{5}{|c|}{272} \\
\hline$R_{B}[N]$ & \multicolumn{5}{|c|}{169} \\
\hline
\end{tabular}

The dissipation power due to the friction in bearings can be viewed in fig. 11 .

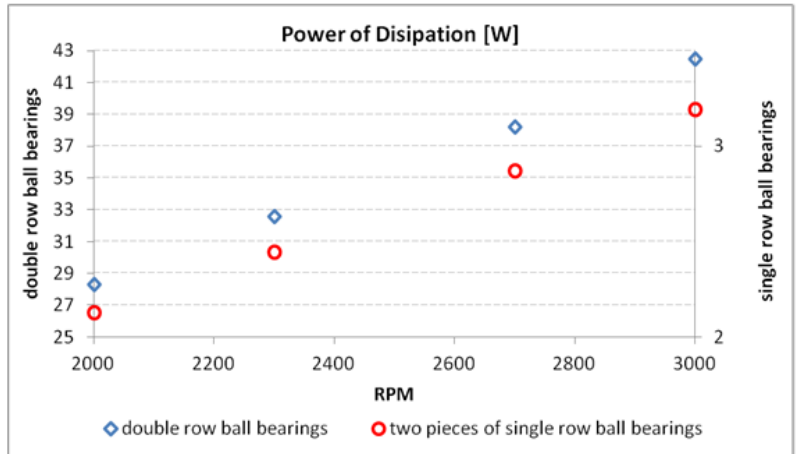

Fig. 11 Dissipation power in bearings

\section{Shaft friction dissipations}

We have derived the relations to calculate the shaft dissipation power from the basic laws of mechanics and fluid mechanics. We will come out of Fig. 12 .

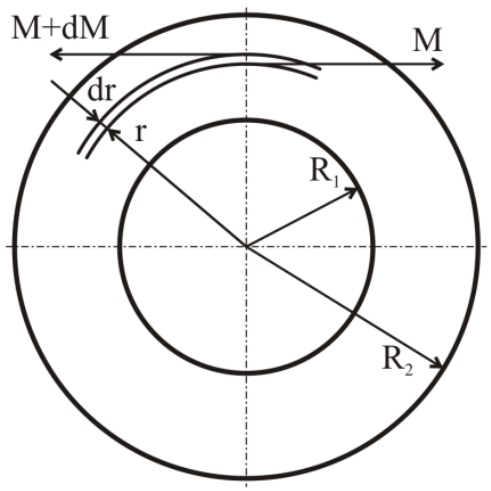

Fig. 12 Scheme for computation

$$
\begin{gathered}
M+d M-M=0 \\
\int d M=\int 0 \Rightarrow M=C_{1} \\
M=F_{f} \cdot r=\tau \cdot S \cdot r=\tau \cdot 2 \cdot \pi \cdot r \cdot L \cdot r \\
\tau=\eta \cdot \frac{d w}{d r} \\
\int_{w_{2}}^{w} d w=\int_{R_{2}}^{r} \frac{d w}{2 \cdot \eta \cdot \pi \cdot L} \cdot \frac{C_{1}}{C_{1}^{2}} \mid w_{2}=0 \\
\left.w=\frac{1}{2 \cdot \pi \cdot \eta \cdot L} \cdot \frac{1}{R_{2}}-\frac{1}{r}\right) \\
C_{1}: r=R_{1} ; w=w_{o} \\
w_{o}=\frac{C_{1}}{2 \cdot \pi \cdot \eta \cdot L} \cdot\left(\frac{1}{R_{2}}-\frac{1}{R_{1}}\right) \\
P_{f s}=M \cdot \omega=w_{o} \cdot 2 \cdot \pi \cdot \eta \cdot L \cdot \frac{R_{1} \cdot R_{2}}{R_{1}-R_{2}} \cdot \omega
\end{gathered}
$$


The resulting relation shall be:

$$
P_{f s}=M \cdot \omega=\frac{8 \cdot v \cdot \rho \cdot \pi^{3} \cdot L \cdot R_{1}^{2} \cdot R_{2} \cdot n^{2}}{R_{1}-R_{2}}
$$

where:

$\begin{array}{ll}M & \text { torsion moment } \\ F_{f} & \text { friction force } \\ \tau & \text { shear stress } \\ \eta & \text { dynamic viscosity } \\ S & \text { shaft surface } \\ v & \text { kinematic viscosity (of air) }\end{array}$

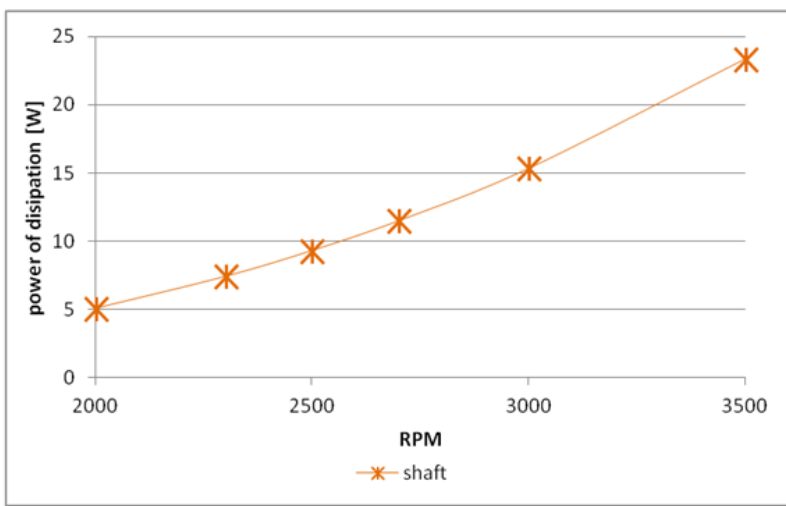

Fig. 13 Shaft dissipation power

\section{Conclusion}

As stated in the introduction, it is necessary to determine the power of rotor dissipation correctly in order to determine the efficiency of the rotating system. This article presents a procedure of dissipation determination on the example of the power of dissipation of a particular rotor (the rotor of the experimental VT-400 turbine). We will have to include also dissipations in the dynamometer into this calculation. However, it is not so simple, therefore there is an option to use a moment flange, due to which the dynamometer losses can be omitted.

The dissipation analysis followed by the power of dissipation determination clearly shows the influence of particular sources of dissipations. (see fig. 13). The highest proportion - almost a half - belongs to losses occured in bearings (at lower speed), mainly in the axial force absorbing bearing (fig. 11), and losses caused by the disc friction in the surrounding environment.

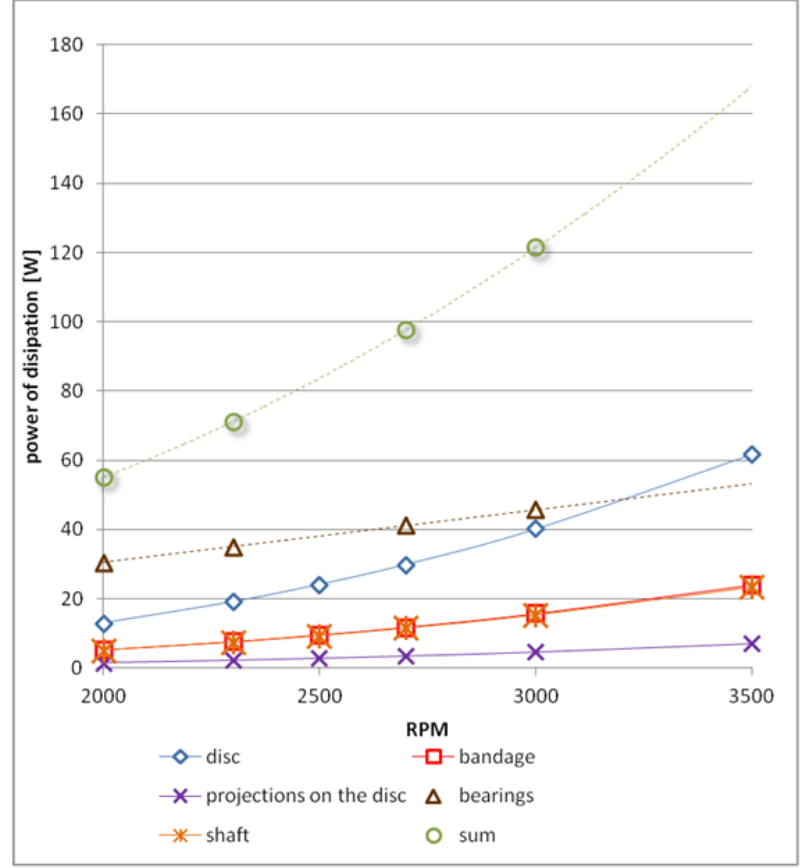

Fig. 14 Rotor dissipation power

As manifested in this article, it is an analytical solution of this matter only and will be followed by an experimental verification of results in the near future. The objective is to find and verify such a methodology which we could use for various types of blade wheels without performing new complex experimental verifications of the dissipation power for every change of operational parameters or blading replacement.

The presented work was financially supported by the Ministry of Education, Youth and Sport Czech Republic Project LQ1603 (Research for SUSEN). This work has been realized within the SUSEN Project (established in the framework of the European Regional Development Fund (ERDF) in project CZ.1.05/2.1.00/03.0108) and student project SGS-2016-045 (Improving the efficiency, reliability and service life of power machines and equipment 4 ).

\section{References}

1. A. Stodola, Dampf u. Gasturbinen, 1924

2. G.A. Zalf, V.V. Zvjagincev Těplovoj rasčet parovych turbin. Mašgiz Moskva, 1961

3. A.V. Ščegljajev, Parovyje turbiny. Energija Moskva, 1967

4. L. Miškovský, Parní a plynové turbíny. Praha, 1949

5. W. Traupel, Termische Turbomaschinen. Springer, 1958

6. E. Tuliszka, Turbiny cieplne. Wydawnictwa Naukovo - Techniczne Warszawa, 1973

7. H. Linnecken, Der Radreibungsverlust insbesondere bei Turbomaschinen. AEG Mitteilungen, 1957

8. [online]. 15.8.2016 [cit. 2016-08-15]. Available on: http://www.elotechnik.cz/lozisko-2217-kzvl.html\#zalozka-2 